1D composite fermions: Bogoliubov-like mode in the Tonks-Girardeau gas

This article has been downloaded from IOPscience. Please scroll down to see the full text article. 2006 Europhys. Lett. 74785

(http://iopscience.iop.org/0295-5075/74/5/785)

View the table of contents for this issue, or go to the journal homepage for more

Download details:

IP Address: 128.97.26.218

The article was downloaded on 27/05/2011 at 00:06

Please note that terms and conditions apply. 
Europhys. Lett., 74 (5), pp. 785-791 (2006)

DOI: $10.1209 / \mathrm{epl} / \mathrm{i} 2006-10034-8$

\title{
1D composite fermions: Bogoliubov-like mode in the Tonks-Girardeau gas
}

\author{
I. V. OvChinnikov and D. Neuhauser \\ Department of Chemistry and Biochemistry, University of California at Los Angeles \\ Los Angeles, CA, 90095-1569, USA
}

received 24 February 2006; accepted 4 April 2006

published online 5 May 2006

PACS. 05.30.Jp - Boson systems.

PACS. 71.10.Pm - Fermions in reduced dimensions (anyons, composite fermions, Luttinger liquid, etc.).

PACS. 71.45.Gm - Exchange, correlation, dielectric and magnetic response functions, plasmons.

\begin{abstract}
We reformulate 1D boson-fermion duality in path-integral terms. The result is a $1 \mathrm{D}$ counterpart of the boson-fermion duality in the 2D Chern-Simons gauge theory. The theory is consistent and enables, using standard resummation techniques, to obtain the longwavelength asymptotics of the collective mode in $1 \mathrm{D}$ boson systems at the Tonks-Girardeau regime. The collective mode has the dispersion of Bogoliubov phonons: $\omega(q)=q \sqrt{\bar{\rho} U(q) / m}$, where $\bar{\rho}$ is the bosons density and $U(q)$ is a Fourier component of the two-body potential.
\end{abstract}

1D boson systems attract much attention [1-20] in light of recent experiments on cigarshaped atomic traps [1-6] and gases exposed to linear carbon nanotubes [7-9]. The history of theoretical studies of 1D bosons goes back to the celebrated work by Lieb and Liniger, who found exact integrability of zero-range interacting bosons via the Bethe ansatz, for all values of the interaction strength $[21,22]$. There are two limiting cases for 1D systems: the high-density weak-interaction Thomas-Fermi (TF) regime [20], where the Bogoliubov energy functional [23, $24]$ and the thermodynamic limit of the Gross-Pitaevskii mean-field theory [25-27] apply; and the low-density strong interaction Tonks-Girardeau (TG) regime of impenetrable bosons [2833]. Unlike the TF regime, in the TG regime the boson wave function is Fermi-like and the fermion-boson duality method has been proposed for this regime [17, 19, 30,31].

The original Lieb-Liniger first-quantization approach enables to find analytically the lowenergy elementary excitations spectrum, i.e., the spectrum of the single particle-hole pair excitations, for all values of interaction constants [34]. However, as first pointed out by Lieb (see sect. III in ref. [34]), within this approach it is not possible to analyze the quasiparticles or collective modes in the system. In the TF regime, the study of the collective modes can be accomplished by using the Gross-Pitaevskii energy functional with the introduction of the classical order parameter field $\left({ }^{1}\right)$. It turns out that in the TF regime the collective mode and the elementary excitations are similar.

$\left({ }^{1}\right)$ See, e.g., ref. [35] and references therein. 
As to the TG regime, there is still no analytical tool to study the collective mode. In this letter we propose an exact path-integral approach based on the boson-fermion duality idea. The method is actually a $1 \mathrm{D}$ analogue of the $2 \mathrm{D}$ composite particles formalism, which enables mapping fermions to bosons and vice versa in 2D by the coupling to the Chern-Simons gauge field $\left({ }^{2}\right)$. As a result, we obtain that far inside the TG regime the situation is reminiscent of that in Fermi systems: the collective mode lies above the elementary excitation spectrum, which is Fermi-like, and the dispersion of the collective mode turns out to be of Bogoliubov form, i.e., sound with velocity proportional to the square root of the interaction constant.

We start from a secondary quantized Hamiltonian of a homogeneous system of spinless $1 \mathrm{D}$ bosons, which interact through a two-body potential $U$ :

$$
\begin{aligned}
\hat{H} & =\hat{K}+\hat{U} \\
\hat{K} & =\frac{1}{2 m} \int \mathrm{d} x \hat{\psi}_{b}^{\dagger}(x)\left(-i \partial_{x}\right)^{2} \hat{\psi}_{b}(x), \\
\hat{U} & =\frac{1}{2} \int \mathrm{d} x \mathrm{~d} x^{\prime} \delta \hat{\rho}_{b}(x) U\left(x-x^{\prime}\right) \delta \hat{\rho}_{b}\left(x^{\prime}\right),
\end{aligned}
$$

where $m$ is the mass; $\partial_{x}=\partial / \partial x ; \hat{\rho}_{b}(x)=\hat{\psi}_{b}^{\dagger} \hat{\psi}_{b}(x)$ with $\hat{\psi}_{b}$ being the boson operators and $\delta \hat{\rho}_{b}(x) \equiv \hat{\rho}_{b}(x)-\bar{\rho}_{b}$ is the density fluctuations where $\bar{\rho}_{b}$ is the average boson spatial density.

The many-particle wave function in the TF regime experiences no crucial changes as one particle passes another, whereas in the TG regime it falls down almost to zero as the coordinate of one particle approaches the position of another one. The "fermionized" boson wave function has zero value if the position of one particle coincides with that of another one. Such a reduction of the Hilbert space to fermionized wave functions is an approximation, but is justified far inside the TG regime.

A fermionized boson wave function can be constructed from a fermion antisymmetric wave function in the following way $[19,30,31]$ :

$$
\psi_{b}\left(\left\{x_{i}\right\}\right)=\prod_{i<j} \operatorname{sign}\left(x_{i}-x_{j}\right) \psi_{f}\left(\left\{x_{i}\right\}\right) .
$$

This relation is an approximation which is justified only far inside the TG regime.

In a secondary-quantized language eq. (1) corresponds to the introduction of the new quasi-particle operators $\hat{\psi}_{f}^{\dagger}$, which are related to $\hat{\psi}_{b}^{\dagger}$ by

$$
\hat{\psi}_{b}^{\dagger}(x)=\hat{\psi}_{f}^{\dagger}(x) \exp \left[-i \pi \int \mathrm{d} x^{\prime} \theta\left(x-x^{\prime}\right) \hat{\rho}\left(x^{\prime}\right)\right],
$$

where $\theta$ is the Heaviside unit step function and

$$
\hat{\rho}(x) \equiv \hat{\psi}_{b}^{\dagger}(x) \hat{\psi}_{b}(x)=\hat{\psi}_{f}^{\dagger}(x) \hat{\psi}_{f}(x)
$$

is the spatial particle density, which has the same form in terms of initial bosons and the new quasi-particles. It is easy to prove that

$$
\hat{\psi}_{f}\left(x_{1}\right) \hat{\psi}_{f}^{\dagger}\left(x_{2}\right)-e^{-i \pi \Delta} \hat{\psi}_{f}^{\dagger}\left(x_{2}\right) \hat{\psi}_{f}\left(x_{1}\right)=\left\{\hat{\psi}_{f}\left(x_{1}\right), \hat{\psi}_{f}^{\dagger}\left(x_{2}\right)\right\}=\delta\left(x_{1}-x_{2}\right),
$$

where $\Delta=\theta\left(x_{1}-x_{2}\right)-\theta\left(x_{2}-x_{1}\right)=\operatorname{sign}\left(x_{1}-x_{2}\right)= \pm 1$. That is, the new operators satisfy Fermi anti-commutation relations so that the quasi-particles are fermions. Let us call them composite fermions $(\mathrm{CF})$, after their predecessors in 2D.

\footnotetext{
$\left({ }^{2}\right)$ For a review see, e.g., ref. [36]. Our approach, however, is closer to that of ref. [37].
} 
To see that the transformation (2) corresponds to eq. (1), note that if one starts creating a boson wave function by repeatedly acting on a vacuum state with the operators $\hat{\psi}_{b}^{\dagger}$ from eq. (2), then the CF operators will produce a fermionic wave function and the exponential phase-factors will give the "sign" term in eq. (1).

The kinetic energy and two-body interaction in the CF operators' representation take the following forms:

$$
\begin{aligned}
\hat{K} & =\frac{1}{2 m} \int \mathrm{d} x \hat{\psi}_{f}^{\dagger}(x)\left(-i \partial_{x}+k_{F}+\hat{a}_{x}\right)^{2} \hat{\psi}_{f}(x), \\
\hat{U} & =\frac{1}{2 \pi^{2}} \int \mathrm{d} x \mathrm{~d} x^{\prime} \hat{a}_{x}(x) U\left(x-x^{\prime}\right) \hat{a}_{x}\left(x^{\prime}\right),
\end{aligned}
$$

with the constraint

$$
\hat{a}_{x}(x)=\pi \delta \hat{\rho}(x)
$$

where $k_{F}=\pi \bar{\rho}$ is the Fermi wave vector.

In a path-integral representation the constraint (3) is easily incorporated with the aid of a Lagrange multiplier. The partition function has the following form so far:

$$
\begin{gathered}
\mathcal{Z}(\phi)=\int \mathcal{D} \psi_{f} \mathcal{D} \psi_{f}^{\dagger} \mathcal{D} a_{x} e^{i \int \mathrm{d} t \mathcal{L}} \prod_{\{t, x\}} \delta\left(\frac{a_{x}}{\pi}-\rho+\bar{\rho}\right), \\
\mathcal{L}=\int \mathrm{d} x\left(\psi_{f}^{*}\left(i \partial_{t}\right) \psi_{f}+\rho \phi\right)-K\left(\psi_{f}^{*}, \psi_{f}\right)-U\left(a_{x}\right),
\end{gathered}
$$

where $\rho \equiv \psi_{f}^{*} \psi_{f}$ and the constrained path integration is over the statistical field $a_{x}$ and the Grassmann fields $\psi_{f}$ and $\psi_{f}^{*}$ which represent the CFs. An external potential $\phi$ has also been added to the action. We will use it to probe the system, i.e., the density-density correlation function is

$$
\left\langle\hat{\rho}(t, x) \hat{\rho}\left(t^{\prime}, x^{\prime}\right)\right\rangle=-\left.\mathcal{Z}(\phi)^{-1} \frac{\delta^{2} \mathcal{Z}(\phi)}{\delta \phi(t, x) \delta \phi\left(t^{\prime}, x^{\prime}\right)}\right|_{\phi=0} .
$$

The constraint in the partition function (4) can be rewritten through the introduction of an auxiliary field $a_{t}$ as

$$
\prod_{\{t, x\}} \delta\left(\frac{a_{t}}{\pi}-\rho+\bar{\rho}\right)=\int \mathcal{D} a_{t}(t, x) e^{-i \int \mathrm{d} t \mathrm{~d} x\left(\frac{a_{x}}{\pi}-\rho+\bar{\rho}\right) a_{t}} .
$$

As a result, the spatial density of the Lagrangian becomes

$$
\begin{aligned}
\mathcal{L}= & \psi_{f}^{*}\left(i \partial_{t}-\frac{\left(-i \partial_{x}+k_{F}+a_{x}\right)^{2}}{2 m}\right) \psi_{f}-a_{t}\left(\frac{a_{x}}{\pi}-\rho+\bar{\rho}\right)+ \\
& +\rho \phi-\frac{1}{2 \pi^{2}} \int \mathrm{d} x^{\prime} a_{x}(x) U\left(x-x^{\prime}\right) a_{x}\left(x^{\prime}\right),
\end{aligned}
$$

and the integration in $\mathcal{Z}$ is assumed now over a time-space statistical field $\boldsymbol{a}=\left(a_{t}, a_{x}\right)$.

Before proceeding further, let us outline the connection of the proposed boson-fermion transformation to existing theories. The transformation (2) is reminiscent of the inverse bosonfermion transformation in Haldane's bosonization approach for 1D Fermi liquids $\left({ }^{3}\right)$. Nevertheless, Haldane's bosonization is developed for studies of low-frequency physics of fermion

$\left({ }^{3}\right)$ For review see, e.g., Chap. 5 of Ref. [38]. 
systems in terms of bosons, which, in fact, represent the spatial density fluctuations of the fermions. In our case, however, the transformation (2) involves operators of real bosons and not density fluctuations. Density fluctuations, instead, are represented by the spatial statistical field component $a_{x}$.

Physically, the proposed model is a 1D counterpart of Jain's mechanism of attaching flux quanta to 2D particles, which leads to the coupling of the composite objects to a ChernSimons gauge field [36]. There are, however, several aspects in which the theory proposed differs from the Chern-Simons theory, apart from the different dimensionalities of the systems. i) The proposed theory is not gauge invariant. ii) Time-reversal and space-reversal symmetries are broken separately, though time-space-reversibility is present. iii) The coupling to ChernSimons gauge fields in 2D results in an additional magnetic field experienced by the composite objects, whereas in our case the coupling to the statistical fields leads to a shift of the oneparticle kinetic energy dispersion by $k_{F}$ in momentum space. In a sense, it looks as though the whole system starts moving. This fact is going to reveal itself later through a Doppler shift in the response function.

The noninteracting part of the action becomes the sum of two Gaussian actions for the CFs and the statistical fields governed by the following "bare" CFs' and statistical fields' propagators, respectively (in Fourier space):

$$
G_{0}^{-1}(\boldsymbol{p})=\varepsilon-\frac{\left(p-k_{F}\right)^{2}}{2 m}, \quad \hat{D}_{0}^{-1}(\boldsymbol{q})=-\frac{1}{\pi}\left[\begin{array}{cc}
0 & 1 \\
1 & v_{F} u(q)
\end{array}\right],
$$

with $u(q) \equiv U(q) /\left(v_{F} \pi\right)$, where $U(q)$ is the spatial Fourier transform of the two-body potential and $\boldsymbol{p}=(\varepsilon, p), \boldsymbol{q}=(\omega, q)$. The interaction part of the action is

$$
\mathcal{L}_{\text {int }}=\left(\phi+a_{t}\right) \rho-a_{x} j
$$

where $j$ is the CF current density:

$$
j=\frac{1}{2 m}\left(\psi_{f}^{*}\left(\left(-i \partial_{x}-k_{F}\right) \psi_{f}\right)+\left(\left(i \partial_{x}-k_{F}\right) \psi_{f}^{*}\right) \psi_{f}+a_{x} \rho\right) .
$$

Due to the coupling to the statistical fields the one-particle dispersion is shifted by $k_{F}$ in momentum space. One can formally make a substitution $p-k_{f} \rightarrow p$ and arrive at the ordinary picture of $1 \mathrm{D}$ fermions at rest.

As the action is Gaussian in the CF fields, one can integrate them out. The integration leads as usual to the fermion determinant in the effective statistical fields' action. At this point it is important to note that so far we have made no approximations beyond the assumption (1). Now in order to obtain the density-density response of the system it suffices to leave in the effective action only the terms quadratic in the statistical fields:

$$
\mathcal{Z}_{\text {eff }}(\phi)=\int \mathcal{D} \tilde{\boldsymbol{a}} \exp \left[i \frac{1}{2} \int \frac{\mathrm{d}^{2} \boldsymbol{q}}{(2 \pi)^{2}} \mathcal{L}_{\text {eff }}\right] .
$$

Here, the effective action has the following form:

$$
\mathcal{L}_{\mathrm{eff}}=\boldsymbol{a}(-\boldsymbol{q})\left(\hat{D}_{0}^{-1}(\boldsymbol{q})-\hat{\Pi}^{D}(\boldsymbol{q})\right) \boldsymbol{a}(\boldsymbol{q})-(\boldsymbol{a}+\phi)(-\boldsymbol{q}) \hat{\Pi}^{P}(q)(\boldsymbol{a}+\phi)(\boldsymbol{q}),
$$

where $\Pi^{D}$ and $\Pi^{P}$ are the diamagnetic and paramagnetic polarization operators, respectively. The renormalized statistical fields' propagator is given by a Dyson equation:

$$
\left.\hat{D}(\boldsymbol{q})=\left(\hat{D}_{0}^{-1}(\boldsymbol{q})-\hat{\Pi}(\boldsymbol{q})\right)\right)^{-1},
$$



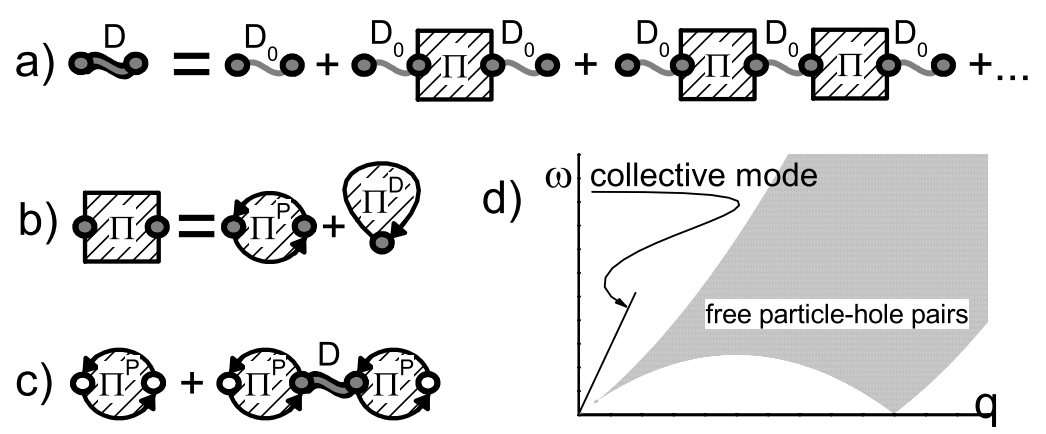

Fig. 1 - (a)-(c) Graphical representation of eqs. (7)-(9). The hollow interaction vertices in (c) denote the density components of the two-component density-current interaction vertex. (d) The excitation spectrum of a $1 \mathrm{D}$ boson system at the TG regime. Besides free particle-hole pair excitations there is a collective mode with a Bogoliubov-like dispersion $\omega(q)=q \sqrt{\bar{\rho} U(q) / m}$.

where

$$
\hat{\Pi}(\boldsymbol{q})=\hat{\Pi}^{P}(\boldsymbol{q})+\hat{\Pi}^{D}(\boldsymbol{q})
$$

is the total polarization operator. Using eq. (5) one can now find the density-density response in the following form (see fig. 1):

$$
\langle\rho(-\boldsymbol{q}) \rho(\boldsymbol{q})\rangle=\left[\hat{\Pi}^{P}(\boldsymbol{q})+\hat{\Pi}^{P}(\boldsymbol{q}) \hat{D}(\boldsymbol{q}) \hat{\Pi}^{P}(\boldsymbol{q})\right]_{t t} .
$$

The advantage of the proposed transformation to CFs is that in normal Fermi liquids $\left({ }^{4}\right)$ we know the exact form of the polarization operator in the long-wavelength limit [39]. Due to the so-called loop-cancellation theorem (see, e.g., ref. [38]), in the long-wavelength limit the polarization operator is given by an RPA expression. In fact, the loop-cancellation theorem is a consequence of gauge invariance, so that one can argue that we can not use it in our case. However, far inside the TG regime (see below) the gauge invariance is restored so that the theorem still applies. In 1D one has $\left(q \ll k_{F}\right)$

$$
\hat{\Pi}^{D}(\boldsymbol{q})=\frac{1}{\pi}\left[\begin{array}{cc}
0 & 0 \\
0 & v_{F}
\end{array}\right], \quad \hat{\Pi}^{P}(\boldsymbol{q})=\frac{1}{\pi} \frac{1}{\omega^{\prime 2}-\left(1-i 0^{+}\right)^{2}}\left[\begin{array}{cc}
v_{F}^{-1} & -\omega^{\prime} \\
-\omega^{\prime} & v_{F}
\end{array}\right],
$$

with $\omega^{\prime}=\omega /\left(v_{F} q\right)$. Substituting these expressions into eq. (9) one gets the density-density response in the long-wavelength limit:

$$
\langle\rho(-\boldsymbol{q}) \rho(\boldsymbol{q})\rangle=\frac{1}{\pi v_{F}\left(\left(\omega^{\prime}-1\right)^{2}-\left(u(q)+2-i 0^{+}\right)\right)} .
$$

As mentioned, we obtained a Doppler shift since the proposed theory lacks time-reversal symmetry, which in turn has its origin in the assumption of fermionized boson wave function (1). (The proposed path-integral composite particle method does not introduce additional approximations.) Note however that, as one goes toward the strong TG regime,

$$
u(q) \gg 1
$$

$\left({ }^{4}\right)$ We assume that the system of the CFs belongs to the class of Fermi liquids with strong forward scattering. The particle-particle and particle-hole instabilities are unlikely to appear in our case because they would have produced a gap in the excitation spectrum, which the initial boson system cannot possess. 
the time-reversal symmetry is restored leading to the following density-density response:

$$
\langle\rho(-\boldsymbol{q}) \rho(\boldsymbol{q})\rangle_{b}=\frac{v_{F}}{\pi} \frac{q^{2}}{\omega^{2}-\left(v(q) q-i 0^{+}\right)^{2}},
$$

where the momentum-dependent "velocity" of the collective mode is

$$
v(q)=\sqrt{\bar{\rho} U(q) / m} .
$$

The fact that we get reasonable results only far inside the TG regime is natural. The fermionization of bosonic wave functions (1) is a good approximation if, and only if, the inter-boson repulsion is very strong, i.e., if the condition (10) is satisfied.

The excitation spectrum of $1 \mathrm{D}$ bosons in the TG regime (see fig. 1d) now appears to consist of two kinds of excitations, just like in Fermi liquids. The first one is elementary excitations corresponding to free particle-hole pairs. The dispersion of these excitations is given by the density of states of the polarization operator $\Pi$. The higher and lower boundaries of the density of states of $\Pi$ are correspondingly the type-I and type-II excitations by Lieb's classification. In the long-wavelength limit elementary excitations are phonons with velocity $v_{F}$. The second one is the collective mode, or quasiparticles, with velocity (11). Though our results are correct only at long-wavelength limit, in analogy with Fermi liquids one can expect that at some momentum the spectrum of the collective mode enters the region of density of states of the elementary excitations. This would mean the end of the collective mode spectrum.

Another issue is the effective two-body interaction, through which the CFs interact. In the limit (10) the gauge invariance is restored and we are left only with density-density interactions in the system, because only the time-time component of the renormalized statistical field propagator survives:

$$
D_{t t}(\boldsymbol{q})=\pi v_{F} u(q)\left(\omega^{\prime 2}-1\right) /\left(\omega^{\prime 2}-u(q)\right) .
$$

In the static limit $\left(\omega^{\prime} \rightarrow 0\right)$ the effective interaction between CFs is $D_{t t}=\pi v_{F}$. This reflects the well-know fact that unlike the initial boson system, in the fermionized case the two-body interaction becomes weak. Notice, however, that in our case with the increase in initial inter-boson potential, $u(q)$, the effective two-body potential between the CFs does not vanish, as in ref. [19], but instead reaches a constant value.

In the limit of high-energy transfer, $\omega^{\prime} \gg u(q)$, we have $D_{t t}(\boldsymbol{q})=U(q)$. This is natural, since at high energies the screening is relatively weak so that the particles interact via the initial potential $U(q)$.

Finally, in the intermediate region, $1<\omega^{\prime}<u(q)$, the so-called overscreening effect takes place and the effective potential becomes attractive. This fact may have interesting consequences.

In conclusion, we propose a path-integral method for statistics transformation in 1D, which leads to the coupling of composite particles to statistical fields, which are representatives of density-current interactions. With the aid of this transformation we have found a longwavelength limit of a collective mode in TG regime of the boson system. The method proposed, however, can be used for further study of TG bosons, e.g., the study of response of the system near $q \sim 2 k_{F}$, the question of the $\mathrm{CDW}$ and BCS stabilities, the effect of statistical field fluctuations on CF propagator, etc. We leave these investigations for a later work.

The 1D fermion-boson duality concept now has an additional supporting argument. If one views the system as bosons, then the collective mode found here is the original Bogoliubov phonons, i.e., with velocity proportional to the square root of the interaction constant. On the other hand, if one views it as fermions, then the collective mode is the Landau zero-sound of a Fermi liquid with short-range interactions. 
This work was supported by the NSF and the PRF.

\section{REFERENCES}

[1] Müller D. et al., Phys. Rev. Lett., 83 (1999) 5194.

[2] Denschlag J., Cassettari D. and Schmiedmayer J., Phys. Rev. Lett., 82 (1999) 2014.

[3] Key M. et al., Phys. Rev. Lett., 84 (2000) 1371.

[4] Arlt J. and Dholakia K., Phys. Rev. A, 63 (2001) 063602.

[5] Bongs K. et al., Phys. Rev. A, 63 (2001) 031602(R).

[6] Görlitz A. et al., Phys. Rev. Lett., 87 (2001) 130402.

[7] Gelb L. D. et al., Rep. Prog. Phys., 62 (1999) 1573.

[8] Evans R., in Fundamentals of Inhomogeneous Fluids, edited by Henderson D. (Dekker, New York) 1992.

[9] Calbi M. M. et al., Rev. Mod. Phys., 73 (2001) 857.

[10] Girardeau M. D. and Ol'shanit M., Phys. Rev. A, 70 (2004) 023608.

[11] Dunjko V., Lorent V. and Ol'shanit M., Phys. Rev. Lett., 86 (2001) 5413.

[12] Girardeau M. D., Nguyen H. and Ol'shanii M., Opt. Commun., 243 (2004) 3.

[13] Schmidt A. G. M., Cheng B. K. and DA Luz M. G. E., Phys. Rev. A, 66 (2002) 062712.

[14] Drummond P. D., Deuar P. and Kheruntsyan K. V., Phys. Rev. Lett., 92 (2004) 040405.

[15] Astrakharchik G. E. et al., Phys. Rev. Lett., 92 (2004) 030402.

[16] Astrakharchik G. E. and Giorgini S., Phys. Rev. A, 68 (2003) 031602(R).

[17] Budde M. and Mölmer K., Phys. Rev. A, 70 (2004) 053618.

[18] Korepin V. E., Bogoliubov N. M. and Izergin A. G., Quantum Inverse Scattering Method and Correlation Functions (Cambridge University Press, Cambridge, UK) 1993.

[19] Cheon T. and Shigehara T., Phys. Rev. Lett., 82 (1999) 2536.

[20] Baym G. and Pethick C. J., Phys. Rev. Lett., 76 (1996) 6.

[21] Lieb E. H. and Liniger W., Phys. Rev., 130 (1963) 1605.

[22] Yang C. N. and Yang C. P., J. Math. Phys., 10 (1969) 1115.

[23] Bogoliubov N. N., J. Phys. (U.S.S.R.), 11 (1947) 23.

[24] Bogoliubov N. N. and Zubarev D. N., Sov. Phys. JETP, 1 (1955) 83.

[25] Gross E. P., Nuovo Cimento, 20 (1961) 454.

[26] Gross E. P., J. Math. Phys. (N.Y.), 4 (1963) 195.

[27] Pitaevskit L. P., Sov. Phys. JeTP, 13 (1961) 451.

[28] Tonks L., Phys. Rev., 50 (955) 1936.

[29] Lenard A., J. Math. Phys., 7 (1966) 1268.

[30] Girardeau M., J. Math. Phys., 1 (1960) 516.

[31] Girardeau M. D. and Wright E. M., Phys. Rev. Lett., 84 (2000) 5691.

[32] Girardeau M. D. and Wright E. M., Phys. Rev. Lett., 84 (2000) 5239.

[33] Das K. K., Girardeau M. D. and Wright E. M., Phys. Rev. Lett., 89 (2002) 170404.

[34] Lieb E. H., Phys. Rev., 130 (1963) 1616.

[35] Kolomeisky E. B., Newman T. J., Straley J. P. and Qi X., Phys. Rev. Lett., 85 (2000) 1146.

[36] Lopez A. and Fradkin E., Phys. Rev. B, 44 (1991) 5246.

[37] Halperin B. I., Lee P. A. and Read N., Phys. Rev. B, 47 (1993) 7312.

[38] Metzner W., Castellani C. and Di Castro C., Adv. Phys., 47 (1998) 317.

[39] Voit J., Rep. Prog. Phys., 57 (1994) 977. 